\title{
SULLA PARTIZIONE DEI NUMERI E SUL NUMERO DEGLI INVARIANTI.
}

\author{
$\mathrm{N} O \mathrm{~T}$ T A
}

\section{DEL PROF. GIUSTO RELLAVITIS.}

Credo utile tener sott'occhio le tavole delle partizioni dei numeri in parti di dato numero e che non superino un dato limite. Io ne faccio l'applicazione alla determinazione del numero degli invarianti delle forme binarie.

§. 1. Teorema. Il numero $B_{\mu}^{(n, p)}$ delle soluzioni delle due equazioni

$$
\alpha_{0}+\alpha_{1} \ldots .+\alpha_{n}=p, \quad n \alpha_{n}+(n-1) \alpha_{n-1} \ldots .+2 \alpha_{2}+\alpha_{1}=\mu
$$

(essendo $\alpha_{0}, \ldots \ldots \alpha_{n}(n+1)$ incognite susceltibili di ogni valore intero positivo, non escluso lo zero) è uguale al numero $B_{\mu}^{(p, n)}$ delle soluzioni del sistema di equazioni

$$
\beta_{0}+\beta_{x} \ldots .+\beta_{p}=n, \quad p \beta_{p}+(p-1) \beta_{p-1} \ldots .+2 \beta_{2}+\beta_{1}=\mu .
$$

Infatti ogni soluzione delle (I) consiste nella partizione del numero $\mu$ in $\alpha_{n}$ numeri eguali ad $n, \ldots \alpha_{2}$ numeri eguali a $2, \alpha_{s}$ eguali ad 1 , ed $\alpha_{0}$ eguali allo zero, essendo $p$ il numero totale delle parti; ed a questa partizione corrisponde come conjugata l'alira

$$
\left(\alpha_{n}+\alpha_{n-1} \ldots .+\alpha_{1}\right)+\left(\alpha_{n}+\alpha_{n-1} \ldots .+\alpha_{2}\right)+\ldots .+\left(\alpha_{n}+\alpha_{n-1}\right)+\alpha_{n}=\mu,
$$

nella quale lo stesso numero $\mu$ è spartito in tutto al più $n$ parti, ognuna delle quali ¿̀ uguale o inferiore a $p$, le quali parti sono quelle indicate con $p,(p-1), \ldots 2,1$ nella seconda delle (II), $i$ loro numeri essendo $\beta_{p}, \ldots \beta_{2}, \beta_{x}$. Così per esempio alla partizione

$$
7.0+6.1+5.0+4.3+3.3+2.0+1.2=29
$$

(ossia 644433311) del numero 29 corrisponde l'altra partizione

$$
9+7+7+4+1+1=29,
$$

ossia $9.1+8.0+7.2+6.0+5.0+4.1+3.0+2.0+1.2=29$.

2. Teorema. I predetti numeri $B_{\mu}^{(n, p)}$ dipendono gli uni dagli altri mediante le relazioni

$$
\boldsymbol{B}_{\mu}^{(n, p)}=\boldsymbol{B}_{\mu}^{(n-1, p)}+\boldsymbol{B}_{\mu-n}^{(n, p-1)},
$$$$
\text { (3) } \quad B_{\mu}^{(n, p)}=B_{\mu}^{(n, p-1)}+B_{\mu-p}^{(n-1, p)} \text {; }
$$

a motivo della (1) $B_{\mu}^{(n, p)}=B_{\mu}^{(p, n)}$ esse sono conseguenze l' una dell' altra : del resto: possono ambedue dimostrarsi rispetto al numero $B_{\mu}^{(n, p)}$ delle partizioni di $\mu$ in $p$ parti 
non superiori ad $n$. Infatli tali partizioni si separano in due parli, cioè quelle che non contengono il numero $n$, il cui numero è per conseguenza $B_{\mu}^{(n-1, p)}$, e quelle che contengono il numero $n$, tolto il quale rimangono le partizioni di $\mu-n$ in $p-1$ parti, il cui numero è $B_{\mu-n}^{(n, p-1)}$, cosi resta dimostrata la (2). Per dimostrare la (3) separiamo tutte le $\boldsymbol{B}_{\mu}^{\left(n_{1} p\right)}$ in due parti, quelle cioè che contengono almeno uno zero (ossia, il cui numero delle parti non supera $p-1$ ) e che sono in numero $B_{\mu}^{n, p-1}$, e quelle che non contengono alcuno zero, e di cui perciò ciascuna parte può diminuirsi d'un'unità, sicchè ne provengono le $B_{\mu-p}^{(n-1, p)}$. Ecco per esempio le 9 partizioni del numero 7 in 5 parti non superiori a 4 , separate in due parti in guisa da dimostrare le predette soluzioni, che in tal caso sono

$$
9=6+3, \quad 9=7+2
$$

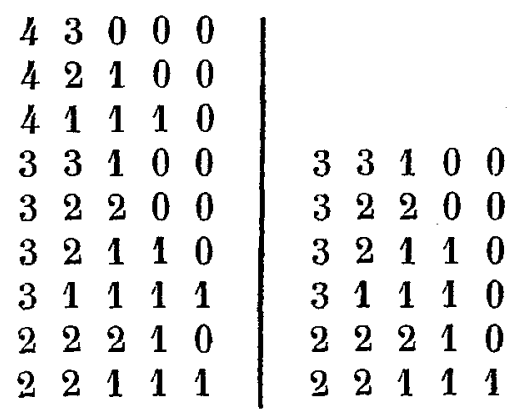

$\begin{array}{llll}3 & 0 & 0 & 0 \\ 2 & 1 & 0 & 0 \\ 1 & 1 & 1 & 0\end{array}$

1. 1110

3. Teorema. E

$$
B_{\mu}^{(n, p)}=B_{n p-\mu}^{(n, p)}
$$

$$
B_{\mu}^{n, p}=0 \text { per } \quad \mu>n p
$$

sicchè c̀ sufficiente conoscere $\mathrm{i}$ valori dei $B$ corrispondenti a $\mu$ non superiori a $\frac{n p}{2}$. La (5) è evidente, e la (4) si dimostra supponendo che a ciascuna delle $p$ parti si sostituisea il suo complemento al numero $n$, nel qual modo la somma $\mu$ diventerà $u p-\mu$.

4. Problema. In quanti modi il mumero $N$ può partirsi in $p$ parti ciascuna delle quali sia uno dei termini della progressione aritmetica $c, c+d, c+2 d \ldots$ $c+n d$ ? Ciascuna delle parti si diminuisca di $c$ poscia si divida per $d$, in tal modo la loro somma diventerà $\frac{N-c p}{d}=\mu$, e perciò il numero cercato sarà $B_{\mu}^{(n, p)}$. Così le partizioni del numero $N=15$ in tre numeri dispari non superiori a 9 sono $B_{6}^{(4,8)}=5$; cioè $951,933,771,753,555$, che dipendono dalle 420, 411, 330, $321,222$.

Corollario. Se rimane indeterminato il numero delle parti, bisogna sommare i valori corrispondenti a $p=1,2,3$, ece. Così per esempio il numero dei modi in cui 
PURA ED APPLICATA.

$\mathrm{N}$ può partirsi neï numeri $2,3,4,5, \ldots .(2+n)$ è

$$
B_{\mathrm{x}-2}^{(n, 1)}+B_{\mathrm{r}-4}^{(n, 2)}+B_{\mathrm{r} \rightarrow 6}^{(n, 3)}+\text { ecc. }
$$

5. Problema. In quanti modi il numero $N$ può partirsi in $p$ parti disuguali scelte nella predetta progressione aritmetica $c, c+d, \ldots c+n d$ ? In ciascuna partizione le parti prese nell'ordine di grandezza crescente si diminuiscano rispettivamente di $c, c+d, c+2 d, \ldots ., c+(p-1) d$, poscia si dividano per $d$, la loro somma diventerà $\frac{N-c p}{d}-\frac{p(p-1)}{2}=\mu$, ed il numero cercato sarà $B_{\mu}^{(n-p+1, p)}$.

Esempio. Volendo partire $N=\mathbf{1 9}$ in tre numeri dispari non superiori a 13, dalle $B_{\mu, p+1, p)}^{(n-p+1}=B_{5}^{(4,3)}=4$ partizioni $410,320,311,221$ dedurremo (raddoppiandone i numeri e sommandovi $5,3,1)$ le $1351,1171,1153,973$.

6. Se il numero $p$ delle parti, nelle quali $\mu$ dee partirsi, è uguale o maggiore di $\mu$ il numero $B_{\mu}^{(n, p)}$ delle partizioni non cangia, noi lo segneremo con $\boldsymbol{B}_{\mu}^{(n)}$. Lo stesso avviene se il numero $n$ è uguale o maggiore di $\mu$; sicchè porremo $B_{\mu}^{(\mu, \mu)}=B_{\mu}^{\left(\mu+1, p_{)}\right.}$ $=$ ecc. $=\boldsymbol{B}_{\mu}^{(\mu)}$. Finalmente se hanno luogo ambedue queste circostanze scriveremo $\boldsymbol{B}_{\mu}$ in luogo di $B_{\mu .}^{(\mu, \mu)}$; questo $B_{\mu} \grave{e}$ dunque il numero delle partizioni di $\mu$ in parti comunque scelte tra i numeri interi, ommettendo di menzionare lo 0 , giacchè ora il numero delle parti è indeterminato. Cosi $B_{4}=5$ esprime il numero delle partizioni 4, $31,22,211,1111$.

7. Mediante la precedente relazione

e cominciando da

$$
B_{\mu}^{(n)}=B_{\mu}^{(n-1)}+B_{\mu-n}^{(n)}
$$

$$
\text { (6) } \quad B_{0}^{(n)}=1, \quad \text { e } \quad(7) \quad B_{\mu}^{(1)}=1
$$

si può calcolare la seguente tavoletta già data dall'Eulero

$$
\begin{array}{ccccccccccccc}
n= & 1 & 2 & 3 & 4 & 5 & 6 & 7 & 8 & 9 & 10 & 11 & 12 \\
\mu=1 & 1 & & & & & & & & & & & \\
2 & 1 & 2 & & & & & & & & & & \\
3 & 1 & 2 & 3 & & & & & & & & & \\
4 & 1 & 3 & 4 & 5 & & & & & & & & \\
5 & 1 & 3 & 5 & 6 & 7 & & & & & & & \\
6 & 1 & 4 & 7 & 9 & 10 & 11 & & & & & & \\
7 & 1 & 4 & 8 & 11 & 13 & 14 & 15 & & & & & \\
8 & 1 & 5 & 10 & 15 & 18 & 20 & 21 & 22 & & & & \\
9 & 1 & 5 & 12 & 18 & 23 & 26 & 28 & 29 & 30 & & & \\
10 & 1 & 6 & 14 & 23 & 30 & 35 & 38 & 40 & 41 & 42 & & \\
11 & 1 & 6 & 16 & 27 & 37 & 44 & 49 & 52 & 54 & 55 & 56 & \\
12 & 1 & 7 & 19 & 34 & 47 & 58 & 65 & 70 & 73 & 75 & 76 & 77
\end{array}
$$


in ciascheduna riga s'intenda ripetuto indefinitivamente l'ultimo numero; cosi per esempio $B_{5}^{(8)}=7$; sicchè i numeri della diagonale sono appunto i $B_{\mu}$.

8. Le altre tavole si calcolano mediante le (2) (3) cominciando da (6) $B_{s}^{(n, p)}=1$, c da (8) $B_{\mu}^{(1, p)}=1$ finchè $\mu$ non supera $p$, e (5) $B_{\mu}^{(1, p)}=0$ quando $\mu$ supera $p$.

\begin{tabular}{|c|c|c|c|c|c|c|c|c|c|c|c|c|c|c|c|}
\hline \multicolumn{2}{|c|}{$p=\varnothing$} & \multicolumn{2}{|c|}{$p=3$} & \multicolumn{3}{|c|}{$p=4$} & \multicolumn{4}{|c|}{$p=5$} & \multicolumn{5}{|c|}{$p=6$} \\
\hline$n=$ & 2 & & & 2 & 3 & 4 & 2 & 3 & 4 & 5 & 2 & 3 & 4 & 5 & 6 \\
\hline$\mu=1$ & 1 & & 1 & 1 & 1 & 1 & 1 & 1 & 1 & 1 & 1 & 1 & 1 & 1 & 1 \\
\hline & 2. & & 2 & 2 & 2 & 2 & 2 & 2 & 2 & 2 & 2 & 2 & 2 & 2 & 2 \\
\hline & 1 & & 3 & 2 & 3 & 3 & 2 & 3 & 3 & 3 & 2 & 3 & 3 & 3 & 3 \\
\hline 4 & 1 & & 3. & 3. & 4 & 5 & 3 & 4 & 5 & 5 & 3 & 4 & 5 & 5 & 5 \\
\hline 5 & 0 & & 3 & 2 & 4 & 5 & 3. & 5 & 6 & 7 & 3 & 5 & 6 & 7 & 7 \\
\hline 6 & & & 3 & 2 & 5. & 7 & 3 & 6 & 8 & 9 & 4. & 7 & 9 & 10 & 11 \\
\hline 7 & & 0 & 2 & 1 & 4 & 7 & 2 & 6. & 9 & 11 & 3 & 7 & 10 & 12 & 13 \\
\hline 8 & & & 1 & 1 & 4 & 8. & 2 & 6 & 11 & 14 & 3 & 8 & 13 & 16 & 18 \\
\hline 9 & & & 1 & 0 & 3 & 7 & 1 & 6 & 11 & 16 & 2 & 8. & 14 & 19 & 22 \\
\hline 10 & & & 0 & & 1 & 7 & 1 & 5 & 12. & 18 & 2 & 8 & 16 & 23 & 28 \\
\hline 11 & & & & & 1 & 5 & 0 & 4 & 11 & 19 & 1 & 7 & 16 & 25 & 32 \\
\hline 12 & & & & & 0 & 5 & & 3 & 11 & 20 & 1 & 7 & 18. & 29 & 39 \\
\hline 13 & & & & & & 3 & & 2 & 9 & 20 & 0 & 5 & 16 & 30 & 42 \\
\hline 14 & & & & & & 2 & & 1 & 8 & 19 & & 4 & 16 & 32 & 48 \\
\hline 15 & & & & & & 1 & & 1 & 6 & 18 & & 3 & 14 & 32. & 51 \\
\hline 16 & & & & & & 1 & & 0 & 5 & 16 & & 2 & 13 & 32 & 55 \\
\hline 11 & & & & & & 0 & & & 3 & 14 & & 1 & 10 & 30 & 55 \\
\hline 18 & & & & & & & & & 2 & 11 & & 1 & 9 & 29 & 58. \\
\hline
\end{tabular}


PURA ED APPLICATA.

\begin{tabular}{ccccccc|ccccccc}
\multicolumn{1}{c|}{$p=7$} & \multicolumn{7}{|c}{$p=8$} \\
\hline$n=$ & 2 & 3 & 4 & 5 & 6 & 7 & 2 & 3 & 4 & 5 & 6 & 7 & 8 \\
7 & 4. & 8 & 11 & 13 & 14 & 15 & 4 & 8 & 11 & 13 & 14 & 15 & 15 \\
8 & 4 & 9 & 14 & 17 & 19 & 20 & 5. & 10 & 15 & 18 & 20 & 21 & 22 \\
9 & 3 & 10 & 16 & 21 & 24 & 26 & 4 & 11 & 17 & 22 & 25 & 27 & 28 \\
10 & 3 & 10 & 19 & 26 & 31 & 34 & 4 & 12 & 21 & 28 & 33 & 36 & 38 \\
11 & 2 & 10 & 20 & 30 & 37 & 42 & 3 & 12 & 23 & 33 & 40 & 45 & 48 \\
12 & 2 & 10 & 23 & 35 & 46 & 53 & 3 & 13. & 27 & 40 & 51 & 58 & 63 \\
13 & 1 & 9 & 23 & 39 & 52 & 63 & 2 & 12 & 28 & 45 & 59 & 70 & 77 \\
14 & 1 & 8 & 24. & 43 & 61 & 75 & 2 & 12 & 31 & 52 & 71 & 86 & 97 \\
15 & 0 & 7 & 23 & 46 & 68 & 87 & 1 & 11 & 31 & 57 & 81 & 101 & 116 \\
16 & & 5 & 23 & 48 & 76 & 100 & 1 & 10 & 33. & 63 & 94 & 120 & 141 \\
17 & 4 & 20 & 49 & 81 & 112 & 0 & 8 & 31 & 66 & 103 & 137 & 154 \\
18 & 3 & 19 & 49 & 88 & 125 & & 7 & 31 & 70 & 116 & 158 & 194 \\
19 & 2 & 16 & 48 & 90 & 136 & & 5 & 28 & 71 & 123 & 176 & 221 \\
20 & 1 & 14 & 46 & 94 & 146 & 4 & 27 & 73 & 134 & 197 & 255 \\
21 & 1 & 11 & 43 & 94 & 155 & & 3 & 33 & 71 & 139 & 214 & 284 \\
22 & 0 & 9 & 39 & 94 & 162 & & 2 & 21 & 70 & 146 & 233 & 319 \\
23 & & 6 & 35 & 88 & 166 & & 1 & 17 & 66 & 147 & 247 & 348 \\
24 & & 5 & 30 & 81 & 169 & 1 & 15 & 63 & 151. & 263 & 383
\end{tabular}

9. Problema. Analogamente al problema del $\$ 6$. si dimandi in quanti modi $A_{\mu}^{(k)}$ il numero $\mu$ può partirsi in parli disuguali $1,2, \ldots . . n$ senza determinare il numero delle parti. Conforme al $\ 5$ diminuiremo le parti ordinatamente di $1,2,3 \ldots$ e siccome il numero delle parti può essere $1,2,3,4 \ldots$. , così la loro somma verrà a diminuirsi di $1,3,6,10$; perciò

$$
A_{\mu}^{(n)}=B_{\mu-1}^{(n-1,1)}+B_{\mu-2}^{(n-2,2)}+B_{\mu-6}^{(n-3,3)}+\text { ecc. }
$$

mediante la (3) due termini successivi possono sommarsi in uno solo, sicchè

$$
A_{\mu}^{(n)}=B_{\mu-1}^{(n-4,2)}+B_{\mu-6}^{(n-3,2)}+B_{\mu-15}^{(n-5,6)}+\text { ecc. }
$$

Per esempio

$$
A_{21}^{(14)}=B_{20}^{(0.2)}+B_{15}^{8.6)}+B_{6}^{(6.6)}=B_{0}+B_{15}^{(8.4)}+B_{6}=1+31+11=43 .
$$

Questi $A_{\mu}^{(n)}$ sono sottoposti alla relazione $A_{\mu}^{(n)}=A_{\mu}^{(n-1)}+A_{\mu-n}^{(n-1)}$ poco differente dalla (2').

10. Numero dei peninvarianti. Io dico peninvarianti di grado $p$ e di indice $\mu$ una funzione intera $P$ delle quantità $a_{0}, a_{1}, a_{2}, \ldots a_{n}$, tale che ogni termine contenga $p$ quantità, $\mathrm{i}$ cui indici abbiaso la somma $\mu$, e sia $\Delta \boldsymbol{P}=0$, dove la ca- 
ratlcristica $\Delta$ indica la derivazione espressa da

$$
a_{0} \mathrm{D}_{a_{1}}+2 a_{1} \mathrm{D}_{a_{2}} \ldots+n a_{n-1} \mathrm{D}_{a_{n}}
$$

ciascuna D designando l'ordinaria derivazione rispelto alla lettera posta abbasso (Veggansi i miei Cenni elementari sui discriminanti, invarianti e covarianti negli Atti dell'I. R. Istituto Veneto per Novembre 1858. Tomo IV pag. 65 e 83 ). Sia per esempio $n=5, p=4, \mu=6$ il numero dei termini del peninvariante sarà $B_{\mu}^{(n, p)}=8$, ed esso avrà la forma

$$
\begin{aligned}
A a_{5} a_{1} a_{0}^{2}+B a_{4} a_{2} a_{0}^{2}+ & C a_{4} a_{1}^{2} a_{0}+D a_{3}^{2} a_{0}^{2}+E a_{3} a_{3} a_{1} a_{0}+F a_{3} a_{1}^{3} \\
& +G a_{2}^{3} a_{0}+H a_{2}^{2} a_{1}^{2}
\end{aligned}
$$

mentre la $\Delta P=0$ conterrà $B_{\mu-1}^{(n, p)} \rightleftharpoons 6$ termini, e sarà

$$
\begin{aligned}
\Delta P= & A a_{5} a_{0}^{3}+(5 A+2 B+2 C) a_{4} a_{x} a_{0}^{2}+(4 B+6 D+E) a_{3} a_{2} a_{0}^{2} \\
& +(4 C+2 E+3 F) a_{3} a_{1}^{2} a_{0}+(3 E+6 G+2 H) a_{2}^{2} a_{1} a_{0} \\
& +(3 F+4 H) a_{2} a_{1}^{2}=0
\end{aligned}
$$

sicchè per determinare 8 coefficienti indeterminati abbiamo 6 equazioni, le quali essendo omogenee danno luggo a due soluzioni essenzialmente differenti (non badandosi ai moltiplicatori comuni); le più semplici sono

$$
\begin{array}{ll}
A=0, B=0, C=0, & D=1, E=-6, F=4, G=4, \quad H=-3 ; \\
A=0, B=1, C=-1, & D=-1, E=2, F=0, G=-1, H=0 .
\end{array}
$$

Potrebbe nascer il dubbio che le 6 equazioni fossero riducibili ad un minor numero, sicchè il numero dei peninvarianti fosse maggior di due; nulladimeno io credo poter asserire che : Il numero dei peninvarianti di grado $p$ e di indice $\mu$ formati colle quantità $a_{1}, a_{2} \ldots a_{n} \grave{e}$ sempre $B_{\mu}^{(n, p)}-B_{\mu-1}^{(n, p)}=E_{\mu}^{(n, p)}$, e fra di essi ve ne sarà un numero $E_{\mu}^{(n-1, p)}$ che non conterranno $a_{n}$, un numero $E_{\mu}^{(n-2, p)}$ senza nè $a_{n} n \dot{e}$ $a_{n-1}$, e cosi in seguito. - Nel caso precedente, essendo $E_{6}^{(4,4)}=7-5=2$, tutti due i peninvarianti sono senza $a_{5}$, ed essendo $E_{6}^{(3,4)}=5-4=1$ uno di essi è anche senza $a_{4}$, appunto come abbiamo trovato. - Col mezzo di relazioni identiche alle (2) (3) e delle $E_{0}^{(n, p)}=1, E_{\mu}^{(n, p)}=E_{n p+1-\mu}^{(n, p)}$ si possono facilmente calcolare le tavole dei numeri $E_{\mu}^{(n, p)}$, e le somme delle loro colonne damno i $B_{\mu}^{(n, p)}$ : ecco le due tavole corrispondenti a $p=9$ ed a $p=10$. 


\begin{tabular}{|c|c|c|c|c|c|c|c|c|c|c|c|c|c|c|c|c|c|c|}
\hline \multirow[b]{2}{*}{$n=$} & \multicolumn{9}{|c|}{$p=9$} & \multicolumn{9}{|c|}{$p=10$} \\
\hline & 2 & 3 & 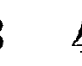 & 45 & 56 & & & & 9 & 2 & 3 & 4 & 5 & 6 & 7 & 8 & 9 & 10 \\
\hline$=0$ & 1 & 1 & 4 & 1 & 1 & & & & 1 & 1 & & 1 & 1 & 1 & 1 & 1 & & 1 \\
\hline & 0 & 0 & ( & 0 & 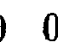 & & & & & & & 0 & 0 & 0 & 0 & & & U \\
\hline 2 & 1 & A & & 1 & 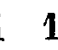 & & & & 1 & & 1 & 1 & 1 & 1 & 1 & & & 1 \\
\hline$\overline{3}$ & 0 & 1 & & & 1 & & & & 1 & 0 & 1 & 1 & 1 & 1 & 1 & & 1 & 1 \\
\hline 4 & 1 & 1 & 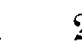 & 8 & 9 & & & & 2 & 1 & 1 & 2 & 2 & 2 & 2 & & 9 & 2 \\
\hline & & 1 & 1 & 2 & 2 & & & & & & 3 & 1 & 2 & 2 & 2 & & & 2 \\
\hline 6 & 1 & 2 & : & 33 & 34 & & & & 4 & 1 & 2 & 3 & 3 & 4 & 4 & 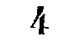 & 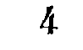 & 4 \\
\hline 7 & 0 & 1 & 2 & 3 & 3 & & & & 4 & 0 & 1 & 2 & 3 & 3 & 4 & 4 & & 4 \\
\hline 8 & & 2 & & 5 & 6 & & & & & & 2 & 4 & 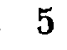 & 6 & 6 & & & $t$ \\
\hline 0 & 0 & 2 & 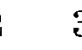 & 35 & ( & & & & 8 & 0 & 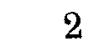 & 3 & 3 & 6 & 7 & & & 8 \\
\hline 1 & 0 & 1 & 4 & 6 & 8 & & & & 10 & 1 & & 5 & & 9 & 10 & 11 & 11 & 12 \\
\hline 1 & -1 & 1 & 3 & 6 & 8 & & & & 12 & -1 & 1 & 3 & 6 & 8 & 10 & 11 & & 12 \\
\hline 12 & a & 1 & 5 & & 1 & & & & 17 & 0 & 2 & 6 & 9 & 13 & 15 & 17 & 18 & 1 \\
\hline 13 & -1 & 0 & 9 & & 10 & & & & 18 & -1 & 0 & 3 & 8 & 11 & 15 & 17 & 19 & 21 \\
\hline & & 0 & 4 & 8 & & & & & 24 & 0 & 1 & 5 & 10 & 16 & 20 & 24 & & 28 \\
\hline 15 & -1 & 0 & 2 & 8 & 1 & & & 3 & 27 & -1 & 0 & 3 & 9 & 15 & 21 & 25 & 29 & 31 \\
\hline 16 & 0 & -1 & 3 & 8 & 16 & & & 9 & 33 & 0 & 0 & 5 & 11 & 19 & 26 & 33 & 37 & 41 \\
\hline & -1 & - & & 7 & 1 & & & & 36 & -1 & -1 & & 9 & 17 & 26 & 33 & & 44 \\
\hline 18 & j & & & & & & & & 44 & 0 & & & & 23 & & & 51 & 5 \\
\hline 19 & & -2 & -2 & & 13 & & & & 46 & -1 & -2 & 0 & 8 & 18 & 32 & 43 & & 61 \\
\hline & 0 & 9 & & j & & & & & 55 & 0 & -1 & & 10 & 24 & 38 & 54 & & 77 \\
\hline$\overline{2}$ & 0 & - & - & 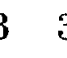 & 8 & & & & 59 & -1 & -2 & -2 & 6 & 19 & 37 & 53 & 70 & 81 \\
\hline 22 & 0 & -2 & - & $2, m$ & 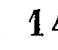 & & & & 6 & 0 & -2 & 0 & 7 & 23 & 42 & 64 & 82 & 100 \\
\hline 23 & 0 & - & & & 0 & & & & 5 & 0 & -2 & -4 & 3 & 17 & 39 & 62 & 36 & 10 \\
\hline 2 & 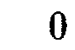 & -1 & & $2-1$ & 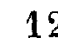 & & & & 7 & 0 & -1 & & , & 22 & 45 & 74 & 101 & 12 \\
\hline
\end{tabular}

11. Applicando il precedente metodo ai peninvarianti, nci quali si lascia indeterminato e il grado $p$ e il numero $n$ delle quantita (il ehe $\dot{c}$ lo stesso come porre $p=n=\mu$ ) io stabilii nel $\$ 9$ della succitata memoria il numero dei peninvarianti di ciascun indice. D'altronde tutti i $B_{\mu}-B_{\mu-x}=E_{\mu}$ peninvarianti d'indice $\mu$. risultano dai peninvarianti fondamentali $P_{2}, P_{3}, P_{4}, \ldots . P_{\mu}$ (che sono i coefficienti della trasformata priva di secondo termine) uno per ciascun indice cominciando dal 2 : il Corollario del $\ 4$ ci dà per questo medesimo numero delle partizioni di $\mu$ nei $2,3, \ldots . \mu$ un'altra espressione; quindi le differenze nella serie $B_{\mathrm{x}}, B_{2}, B_{3} \ldots$ sono date da 


$$
B_{\mu}-B_{\mu-1}=E_{\mu}=B_{\mu-2}^{(1)}+B_{\mu-4}^{(2)}+B_{\mu-6}^{(3)}+\text { ecc. }
$$

12. Forse non è dimostrato, pure può asserirsi con tulta confidenza, che se $\mu=\frac{n p}{2}$ la funzione $\boldsymbol{P}$ soddisfacente alla $\Delta \boldsymbol{P}=\mathbf{0}$ è euritmica rispetto alle quantità $a_{0}, a_{1}, \ldots . a_{n-1}, a_{n} ;$ e perciò essa è ciò che dicesi un invariante. Dunque $(\$ 10)$ : Il numero degli invarianti di grado $p \grave{e} B_{\frac{n p}{2}}^{(n, p)}-B_{\frac{n p}{2}-1}^{(n, p)}$, che per brevilà segneremo con $E^{(n, p)}$.

13. Cerchiamo tulti gl'invarianti della forma biquadratica, cioè sia $n=4$. Essendo

$$
E^{(4,2)}=B_{4}^{(4,2)}-B_{3}^{(4,2)}=3-2=1
$$

si ha un invariante di 3 termini, ehe è il noto $I_{4}^{(2)}=I$ (Veggasi il $\$$ 5. dei Cenni), Poscia $E^{(4,3)}=5-4=1$ c'indica l'invariante di 5 termini $I_{4}^{(3)}=J$. Le

$$
\begin{gathered}
E^{(4,4)}=8-7=1, \quad E^{(4,5)}=12-11=1, \quad E^{(4,6)}=18-16=2, \\
E^{(4,7)}=24-23=1, \quad E^{(4,8)}=33-31=2, \quad E^{(4,9)}=2, \\
E^{(4,10)}=2, \quad E^{(4,1)}=2, \quad E^{(4,12)}=3, \text { ecc. }
\end{gathered}
$$

ci mostrano che dev'esservi anche un invariante di $4^{\circ}$ grado, uno di $5^{\circ}$, due di $6^{\circ}$, ece. Ora pel Corollario del $\$ 4$. coi due invarianti di $2^{\circ}$ e di $3^{\circ}$ grado si possono formare $B_{p-2}^{(1,1)}+B_{p-4}^{(1,2)}+B_{p-6}^{(1,3)}+$ ecc. invarianti di $p^{\text {esimo }}$ grado, e questi numeri

$$
\begin{aligned}
& B_{4-2}^{(1,1)}+B_{4-4}^{(1,2)}=0+1=1, \quad 0+1=1,0+1+1=2, \\
& 0+0+1=1, \quad 0+0+1+1=2,0+0+1+1=2, \\
& 0+0+0+1+1=2,0+0+0+1+1=2, \\
& 0+0+0+1+1+1=3, \text { ecc. }
\end{aligned}
$$

sono appunto $\mathrm{i}$ precedenti; dunque non vi è alcun altro invariante primitivo oltre $\mathrm{i}$ due $I, J$.

14. Per la forma di $5^{\circ}$ ordine. cioè quando $n=5$, le $E^{(5,2)}=0, E^{(5,6)}=0$, ecc. indicano l'assenza d'invariante di grado imparamente - pari (il che ha luogo ogni qual volta $n$ sia dispari). Si ha poi

$$
E^{(5,4)}=12-11=1, \quad E^{(5.8)}=73-71=2, E^{(5,12)}=252-249=3 \text {, }
$$

il che mostra la presenza dei tre invarianti; l'uno $I_{5}^{(5)}$ ha appunto 12 termini (Veggasi Cenni $₫ 6$ ) l'altro $I_{5}^{(8)}$, che il Sig. Faà di Bruno disse primilivo (Annali 1856, pag. 86) dev'essere corretto come segue: 
PURA ED APPLICATA.

$a^{3} c d f^{3}-a^{2} b^{2} d f^{3}-3 a^{2} b c^{2} f^{3}+5 a b^{3} c f^{3}-2 b^{5} f^{3}-a^{3} c e^{2} f^{2}+a^{2} b^{2} e^{2} f^{2}-3 a^{3} d^{2} e f^{2}+$ $+11 a^{2} b c d e f^{2}-5 a b^{3} d e f^{2}+12 a^{2} c^{3} e f^{2}-30 a b^{2} c^{2} e f^{2}+15 b^{4} c e f^{2}+12 a^{2} b d^{3} f^{2}-$ $-21 a^{2} c^{2} d^{2} f^{2}-34 a b^{2} c d^{2} f^{2}+22 b^{4} d^{2} f^{2}+78 a b c^{3} d f^{2}-48 b^{3} c^{2} d f^{2}-27 a c^{5} f^{2}+$ $+18 b^{2} c^{4} f^{2}+5 a^{3} d e^{3} f-5 a^{2} b c e^{3} f-30 a^{3} b d^{2} e^{2} f-34 a^{2} c^{2} d e^{2} f+133 a b^{2} c d e^{2} f-$ $-54 b^{4} d e^{2} f-18 a b c^{3} e^{2} f+3 b^{3} c^{2} e^{2} f+78 a^{2} c d^{3} e f-18 a b^{2} d^{3} e f-220 a b c^{2} d^{2} e f+$ $+106 b^{3} c d^{3} e f+93 a c^{4} d e f-30 b^{2} c^{3} d e f-9 b c^{5} e f-27 a^{2} d^{5} f+93 a b c d^{4} f-$

$-38 b^{3} d^{4} f-42 a c^{3} d^{3} f+8 b^{4} c^{2} d^{3} f+6 b c^{4} d^{2} f-2 a^{3} e^{5}+15 a^{2} b d e^{4}+22 a^{2} c^{2} e^{4}-$

$-54 a b^{2} c e^{4}+27 b^{4} e^{4}-48 a^{2} c d^{2} e^{3}+3 a b^{2} d^{3} e^{3}+106 a b c^{2} d e^{3}-81 b^{3} c d e^{3}-$

$-38 a c^{4} e^{3}+38 b^{2} c^{3} e^{3}+18 a^{2} d^{4} e^{2}-30 a b c d^{3} e^{2}+38 b^{3} d^{3} e^{2}+8 a c^{3} d^{2} e^{2}+$ $+25 b^{2} c^{2} d^{2} e^{2}-57 b c^{4} d e^{2}+18 c^{6} e^{2}-9 a b d^{5} e+6 a c^{2} d^{4} e-57 b^{2} c d^{4} e+74 b c^{3} d^{3} e-$ $-24 c^{5} d^{2} e+18 b^{2} d^{6}-24 b c^{2} d^{5}+8 c^{4} d^{4}$

esso è il più comodo da trovarsi, ed ha 68 termini, mentre il discriminante

$$
\mathrm{D}_{5}=\left(I_{5}^{(4)}\right)^{2}-128 I_{5}^{(8)}
$$

ne ha soltanto 59. Il $I_{5}^{(2)}$ calcolato dal predetto autore ha 232 termini, ma non si può garantire l'esattezza.

15. Se $n=6$ le $E^{(6,1)}=0, E^{(6,3)}=0$, ecc. mostrano che non vi sono invarianti di grado dispari (ciò vale ogni qual volta $n$ sia imparamente - pari). Poscia

$$
\begin{aligned}
& E^{(6,2)}=4-3=1, \quad E^{(6,4)}=18-16=2, \quad E^{(6,6)}=58-55=3, \\
& E^{(6,8)}=151-147=4, \quad E^{(6,10)}=338-332=6, \quad E^{(6,12)}=8, \text { ec. }
\end{aligned}
$$

gli invarianti primitivi $I_{6}^{(2)}$ di 4 termini, $I_{6}^{(4)}$ di 16 termini, e $I_{6}^{(6)}$ danno i seguenti numeri d'invarianti

$$
B_{1}^{(3)}=1, \quad B_{2}^{(2)}=2, \quad B_{3}^{(3)}=3, \quad B_{4}^{(3)}=4, \quad B_{5}^{(3)}=5, \quad B_{6}^{(3)}=7 \text {, eеc.; }
$$

perciò vi dev'essere un invariante primitivo di $10^{\mathrm{m}}$. grado, il quale sarà quasi per certo il discriminante $\mathbf{D}_{6}$. Ritengo per vero che gli invarianti primitivi sieno tra loro indipendenti anche col mezzo delle loro potenze.

16. Quando $n=8$ si ha

$$
\begin{gathered}
E^{(8,2)}=5-4=1, \quad E^{(8,3)}=13-12=1, \quad E^{(8,4)}=33-31=2, \\
E^{(8,5)}=73-71=2, \quad E^{(8,6)}=151-147=4, \quad E^{(8,7)}=289-285=4, \\
E^{(8,8)}=526-519=7, \quad E^{(8,9)}=910-902=8, \\
E^{(8,10)}=1514-1502=12, \quad E^{(8,11)}=13, \quad E^{(8,12)}=20
\end{gathered}
$$

Tom. II. N. 3. 1959. 
sicchè vi $\grave{e}$ un invariante primitivo per ciascuno dei gradi $2,-\ldots .9,10$; infalti (veggasi il Corollario del $\int 4$ ) $B_{p-2}^{(8,1)}+B_{p \rightarrow 4}^{(8,2)}+B_{p \rightarrow 6}^{(8,3)}+$ ecc. dà i numeri

$1,1,1+1=2,1+1=2,1+2+1=4,1+2+1=4,1+3+2+1=7$,

$$
\begin{aligned}
1+3+3+1 & =8, \quad 1+4+4+2+1=12, \\
0+4+5+3+1 & =13, \quad 0+5+7+5+2+1=20,
\end{aligned}
$$

che sono appunto i numeri precedenti. Continuando i calcoli si riconosce l' esistenza anche di un invariante primitivo di $14^{\circ}$ grado; forse esso sarà il discriminante.

17. Gli invarianti primitivi per $n=7$ sono uno di $4^{\circ}$ grado, tre di $8^{\circ}$, ed altri 6 di $12^{\circ}$ grado ecc. - Per $n=9$ sono due di $4^{\circ}$ grado, altri 5 di $8^{\circ}$, ecc. Per $n=10$ sono uno di $2^{\circ}$ grado, uno di $4^{\circ}, 4$ di $6^{\circ}, 5$ di $8^{\circ}$, ece. - Per $n=11$ sono due di $4^{\circ}, 10$ di $8^{\circ}$, ecc. - Per $n=12$ sono uno di $2^{\circ}$, uno di $3^{\circ}$, due di $4^{\circ}$, due di $5^{\circ}, 5$ di $6^{\circ}, 5$ di $7^{\circ}, 8$ di $8^{\circ}$, ec. - Per $n=13$ sono due di $4^{\circ}$, ecc. - Per $n=14$ sono uno di $2^{\circ}$, due di $44^{\circ}$, ecc.

18. Quanti sono $i$ covarianti primitivi della forma cubica ? Se $p$ è il grado e p. l'indice del primo coefficiente $v^{(p, m)}$ del covariante $V^{(m, p)}$ d'ordine $m$ si ha (Cenni ec. $\left.\int 14\right) m=3 p-2 \mu$ e il massimo indice contenuto in $v^{\langle m, p\rangle} \dot{\mathrm{e}} 3$. Il numero dei peninvarianti $v^{(p, m)}$ è dato $(\$ 10)$ da $E_{\mu}^{(n, p)}$, ora si ha $E_{2}^{(3.2)}=1$, e siccome anche $E_{2}^{(2,2)}=1$, cosi il primo covariante è dato da

$$
v^{(2,2)}=a_{2} a_{0}-a_{1}^{2} .
$$

Poscia $E_{3}^{(3,3)}=1$ conduce a trovare

$$
v^{(3,3)}=a_{3} a_{0}^{2}-3 a_{2} a_{\mathrm{i}} a_{\mathrm{o}}+2 a_{1}^{3} .
$$

Similmente $E_{4}^{(3,4)}=E_{4}^{(2,4)}=1$ indica l'esistenza di un $v^{(4 ; 4)}$ senza $a_{3}$, il quale non può essere altro che $\left(v^{(2,2)}\right)^{2}$, ed anche il covariante sarà $\left(V^{(2,2)}\right)^{2}$, cioè non primitivo. La $E_{5}^{(3,5)}=1$ dà un $v^{(5,5)}$ che è il prodotto $v^{(2,2)} \cdot v^{(3,3)}$. Abbiamo $E_{6}^{(3,6)}=2$ e perciò due peninvarianti, uno dei quali sarà compreso in $E_{6}^{(3,5)}=E_{6}^{(3,4)}=1$, e l'altro in $E_{6}^{(2,6)}=1$, e perciò avremo un $v^{(4,0)}$, che è il discriminante, ed un $v^{(6,6)}$ che è il $\left(v^{(2,2)}\right)^{3}$. La $E_{7}^{(3,7)}=8-7=1$ indica il $v^{(7,7)}=\left(v^{(2,2)}\right)^{2}\left(v^{(3,3)}\right)$. La $E_{8}^{(3,8)}=2$ si riduce alle due

$$
E_{8}^{(3.6)}=8-7=1, \quad E_{8}^{(2.8)}=5-4=1,
$$

che mostrano l'esistenza di un peninvariante

$$
v^{(6,2)}=v^{(4,0)} \cdot v^{(2,2)}, \text { e di un } v^{(3,8)}=\left(v^{(2,2)}\right)^{4} \text {. }
$$

Così abbiamo trovato $v^{(2,2)}, v^{(3,3)}$ ed il discriminarte $v^{(4,0)}$, e credo che tutti gli altri dipendano dai loro prodotli, peraltro noi possiamo moltiplicarli per $a_{0}^{i}$, il che 
ne accresce il grado di $i$ e non ne muta l'indice $\mu$; dunque arremo pel primo coefficiente di ogni covariante della forma cubica le tre formule

$$
a_{0}^{i} v^{(2,2)}=v^{(2+i, z+3 i)}, \quad a_{0}^{i} v^{(3,3)}=v^{(3+i, 3+3 i)}, \quad a_{0}^{i} v^{(1,0)}=v^{(4+i, 3 i)},
$$

l'ultima delle quali è esprimibile col mezzo del cubo della prima e del quadrato della seconda.

\section{NOTA}

Ad ogni studioso della matematica si mostra la difficoltà ognora crescente di conoscere ciò che fecero gli altri; per supplire in qualche modo alla mancanza di opere generali, ed anche di repertorii od indici dei Giornali e degli Atti delle Accademie, io proporrei uno spediente. Ognuno che tratta un argomento indichi tutti gli Autori, che, per quanto egli ricorda, hanno pubblicato intorno al medesimo ; chiunque altro scriva dello stesso oggetto ripeta quelle indicazioni aggiungendo le altre, che avrà potuto raccogliere; cosl continuando si potrà sperare che si mantenga la memoria di quanto fu trovato su ciascun argomento. Accompagno al consiglio l'esempio riguardo alla partizione dei numeri. - Eulero, Introd. in anal. infin. I. $\$ 297$. - N. Comm. Acad. Petropolitanae T. III. 1750 pag. 15, 125, et T. XIV. 1769 pag. 168. - Paoli, M. Soc. Italiana 1784. II. pag. 787. - Petri Pauli Liburnensis Opusculum II. - Brunacci, Matem. subl. I \$ 108. Comp. del Calc. subl. 1811. \$ 114. - Lacroix, Traité Calc. diff. et int. 1819. III. $\$ 1193$. - Legendre, Th. des Nombres 1830. II. pag. 128. - Brianchon, J. Ec. polyt. 1837. XXV. pag. 166. - Catalan, J. Liouv, 1838 III. pag. 111. - Rodrigues, J. Liouv. 1839. IV. p. 236. - Jacob, J. Crelle 1846. XXXII. p. 164. - Stern, J. Crelle 1840 XXI p. 01, 177. - Sylvester, Ann. Tortol. 1857. VIII p. 12. - Quarterly, J. 1855 p. 141. - Brioschi, A. Tortol. 1857. VIII. p. 5. - Cayley, A. Tortol. 1858 l. pag. 323.

$$
\text { Padova, Marzo } 1859 .
$$

